\title{
Chronic pelvic pain due to Mycobacterium parascrofulaceum in an Iranian patient: first report of isolation and molecular characterization from Asia
}

\section{Authors}

Hasan Shojaei ${ }^{1}$

Abdolrazagh Hashemi ${ }^{2}$

Parvin Heidarieh ${ }^{3}$

Abass Daei-Naser ${ }^{4}$

${ }^{1}$ Dr, Associate Professor; $\mathrm{PhD}$ in Molecular

Microbiology, Department

of Microbiology, School

of Medicine, Isfahan

University of Medical

Sciences, Isfahan, Iran

${ }^{2}$ Dr., Research Associate,

Infectious Diseases and

Tropical Medicine Research

Center, Isfahan University

of Medical Sciences,

Isfahan, Iran

${ }^{3}$ Dr, Research Associate,

Microbiology Group,

Department of

Pathobiology, School of

Public Health, Tehran

University of Medical

Sciences, Tehran, Iran

${ }^{4}$ Research Assistant;

Infectious Diseases and

Tropical Medicine Research

Center, Isfahan University

of Medical Sciences,

Isfahan, Iran

Submitted on: 11/23/2010 Approved on: 12/28/2010

Correspondence to:

Hasan Shojaei

Hezarjarib street,

Department of

Microbiology,

School of Medicine,

Isfahan University of

Medical Sciences,

Isfahan, Iran

Phone: +98-311-7922409

Fax: +98-311-6688597

hasanshojaei@msn.com

Financial Support:

The current study was

financially supported

by the Office of Vice-

chancellor for Research

of Isfahan University of

Medical Sciences.

We declare no conflict of interest.
Herein we report a case of chronic pelvic pain (CPP) caused by a rare, recently characterized species, $M$. parascrofulaceum, from an Iranian middle aged woman.

\section{CASE REPORT}

A 38 year old woman was admitted to the hospital because of CPP for over three months. There was no apparent evidence of immunodeficiency or HIV infection. In the initial evaluation the urine culture for bacteria was sterile. She was empirically started on antibiotic treatment for the presumed chlamydial or mycoplasmal infection but no improvement was observed. The patient returned to the hospital due to increasing lower abdominal pain. Examination of vaginal discharge confirmed the presence of acid-fast bacilli. The etiologic agent was assumed to be M. tuberculosis and the patient was put on antituberculosis therapy. Two months thereafter due to an aggregated condition the patient underwent hysterectomy.

Strain M79 was isolated from the removed uterus and confirmed by a combination of conventional and molecular tests including the sequence analyses of $16 \mathrm{~S}$ rDNA, hsp65, rpoB, and ITS. ${ }^{1-3}$ The GenBank accession numbers of the isolate determined in this work are as follow: GU121551, HM229794, HM229795 and HM229796 for the $16 \mathrm{~S}$ rDNA, ITS, hsp65, rpoB genes respectively.

Conventional microbiological analysis of the isolate revealed a slowly growing yellow pigmented scotochromogenic mycobacterial species. It was positive for catalase and urease tests and negative for tellurite, nitrate, tween hydrolysis, niacin, arylsulfatase and iron uptake tests. It was suscepti- ble to amikacin, clarithromycin, rifampicin, doxycycline, sulfamethoxazole, streptomycin, and imipenem.

The almost complete $16 \mathrm{~S}$ rDNA gene sequence $(1,426 \mathrm{bp})$ of the isolate showed a $99.58 \%$ similarity with $M$. parascrofulaceum. The signature nucleotide sequences of the isolate showed only a base pair difference at position 250 compared to that of M. parascrofulaceum type strain (Table 1). The ITS, $h s p 65$ and $r p o B$ gene sequences of the isolate showed $98.71 \%, 99.39 \%$ and $100 \%$ similarities with those of the reference strain of $M$. parascrofulaceum, respectively.

In 2004, Turenne et al. introduced $M$. parascrofulaceum as a distinct species. ${ }^{3}$ Later the organism was isolated from a few clinical samples. ${ }^{4}$ However, to the best of our knowledge, there have been no reports from the rest of the world.

Genitourinary infection (GUI) due to non-tuberculous mycobacteria (NTM) is very rare and infrequently reported. ${ }^{5}$ In the Iranian case herein reported M. parascrofulaceum was isolated from a female patient with no apparent immunodeficiency, although she was ill-famed in her community for being a woman with high-risk sexual behavior. Given that most NTM strains are resistant to traditional antituberculous agents, it is important to correctly differentiate genitourinary infections caused by NTM from genitourinary TB. ${ }^{5}$ The clinical management of CPP of this Iranian patient was almost ineffective due misdiagnosis of the causative agent during the initial stages of her infection. This was why the practicing physician eventually resorted to surgical operation.

In conclusion, unless the causative organism is correctly identified noninvasive treatment of such complex cases is unlikely to occur. 
Table 1. Alignment of selected stretches of 16SrDNA gene of Iranian strain of M79 with those of closely related mycobacteria

\begin{tabular}{|c|c|c|c|c|c|c|c|c|c|c|c|c|c|c|c|c|c|c|}
\hline \multirow{2}{*}{ Strain } & \multirow{2}{*}{$\begin{array}{c}\text { GenBank } \\
\text { accession } \\
\text { number }\end{array}$} & \multicolumn{17}{|c|}{ 16S rDNA positions according to E.coli numbering system } \\
\hline & & 128 & 129 & 129 & 134 & 183 & 183 & 183 & 183 & 183 & 183 & 156 & 193 & 193 & 190 & 191 & 250 & 264 \\
\hline M. parascrofulaceum & AF337273 & G & $\mathrm{C}$ & A & G & G & A & $\mathrm{C}$ & $\mathrm{T}$ & $\mathrm{T}$ & G & $\mathrm{C}$ & $\mathrm{C}$ & $\mathrm{C}$ & G & G & A & $\mathrm{T}$ \\
\hline M. palustre & AJ308603 & . & $\mathrm{T}$ & . & . & . & $\mathrm{T}$ & $\mathrm{T}$ & . & A & . & . & . & . & $\mathrm{T}$ & . & $\mathrm{T}$ & $\mathrm{C}$ \\
\hline M. kubicae & AF133902 & . & $\mathrm{T}$ & G & A & . & . & $\mathrm{T}$ & G & A & A & $\mathrm{T}$ & $\mathrm{T}$ & . & A & . & $\mathrm{T}$ & $\mathrm{C}$ \\
\hline M. lentiflavium & AF480583 & . & $\mathrm{T}$ & . & . & . & $\mathrm{T}$ & $\mathrm{T}$ & . & . & . & . & . & . & $\mathrm{T}$ & . & $\mathrm{T}$ & $\mathrm{C}$ \\
\hline M. simiae & X52931 & & $\mathrm{T}$ & . & . & . & . & . & . & . & . & . & . & . & . & . & $\mathrm{T}$ & $\mathrm{C}$ \\
\hline M. scrofulaceum & AF480604 & . & . & . & . & . & . & . & . & . & . & . & . & . & . & . & . & . \\
\hline M 79 & GU121551 & . & . & . & . & . & . & . & . & . & . & . & . & . & . & . & $\mathrm{T}$ & . \\
\hline
\end{tabular}

“.” Indicates that the base pair was identical to that of type strain of M. parascrofulaceum.

\section{ACKNOWLEDGMENTS}

The authors are grateful to the Office of Vice-chancellor for Research of Isfahan University of Medical Sciences for financial support of the current study.

[Braz J Infect Dis 2011;15(2):186-187]@Elsevier Editora Ltda.

\section{REFERENCES}

1. Shojaei H, Magee JG, Freeman R et al. Mycobacterium elephantis sp. nov., a rapidly growing non-chromogenic Mycobacterium isolated from an elephant. Int J Syst Evol Microbiol. 2000; 50 Pt 5:1817-1820.

2. Adekambi T, Colson P, Drancourt M. rpoB-based identification of nonpigmented and late-pigmenting rapidly growing mycobacteria. J Clin Microbiol 2003; 41:5699-5708.

3. Turenne CY, Cook VJ, Burdz TV et al. Mycobacterium parascrofulaceum sp. nov., novel slowly growing, scotochromogenic clinical isolates related to Mycobacterium simiae. Int J Syst Evol Microbiol. 2004; 54:1543-1551.

4. Tortoli E, Chianura L, Fabbro L et al. Infections due to the newly described species Mycobacterium parascrofulaceum. J Clin Microbiol. 2005; 43:4286-4287.

5. Huang CT, Chen CY, Chen HY et al. Genitourinary infections caused by nontuberculous mycobacteria at a university hospital in Taiwan, 1996-2008. Clin Microbiol Infect. 2010;10.1111/ j.1469-0691. 03180.x. 